\title{
Adaptación del currículo de un alumno en el aula regular ${ }^{1}$
}

Cindy Lorena Rodríguez

Universidad Minuto de Dios, UNIMINUTO, Bogotá, Colombia ${ }^{2}$

crodriguezp159@gmail.com

1 Artículo de investigación derivado del trabajo realizado para la tesis de grado en Pedagogía Infantil

2 Licenciatura en Pedagogía Infantil 


\title{
Adaptación del currículo de un alumno en el aula regular
}

\section{Resumen}

Este artículo está basado en la investigación, Adaptación del currículo de un alumno en el aula regular, el cual se fundamenta en la educación inclusiva con habilidades diversas, realizada entre los años 2010-2011 en el Colegio Real de los Andes. La investigación se funda en la experiencia personal de la autora al interior del aula regular, motivada por el deseo de saber cómo se pueden llegar a desempeñar las personas cn habilidades diversas, las cuales deben ser vistas incluidas por la sociedad y más cuando se habla de formación integral, teniendo presente que cada ser humano es único e irrepetible, con potencialidades diversas, grandes expectativas y sueños. Lo anterior implica que el alumno sea el actor principal de un proyecto de vida digno, que servirá para el desarrollo pleno de su personalidad y fortalecerá sus habilidades de tipo académico. En este medio académico es muy importante que la tolerancia sea una cualidad primordial de los educadores, y que no se quede solo a un nivel conceptual. El profesor debe ser, un ser humano con una gran riqueza de valores que se debe proyectar en una pedagogía con mentalidad abierta, libre de prejuicios y preconceptos, para así llegar a poner en práctica su pedagogía.

Palabras clave: Inclusión en el aula, habilidades diversas, formación integral, Pedagogía de la Integración, Colegio Real de los Andes, marco legal, integración.

\section{Adapting the curriculum of a student in the regular classroom}

\begin{abstract}
This article is based on a research, adapting the curriculum of a student in the regular classroom, based on a multi-skilled inclusive education whose data was collected between 2010 and 2011 from Colegio Real de los Andes. The study was based on the author's personal experience with student population inside their regular classroom activities. The author was motivated by the desire to know how one could contribute to society's expectations on an inclusive and integrated education that takes into account the human being as a unique being endowed with different potentials, great expectations, and dreams that nurture him or her into a major player in his or her dignified project of life that will, in turn, contribute towards their full personality growth and hence strengthen their academic skills. Similarly, this will be of great value towards commitment and devotion for inclusion, construed as a paramount import to educational formation. Hence, the dedication of educators in this inclusivity is a fundamental feature not only from the conceptual point of view, but more importantly, as a fundamental element in the essence of an educator, which must be, a human being formed in the richness of values openly projected on a pedagogy without any prejudice and preconceptions during a pedagogical dispensation.
\end{abstract}

Keywords: inclusion, diverse skills, comprehensive training, Educational Integration, Colegio Real de los Andes, legal framework integration.

\section{Adaptação do currículo de um aluno no ensino regulamentar}

\section{Resumo}

Este Artigo está baseado na pesquisa titulada sobre a adaptação do currículo de um aluno no ensino regulamentar, o qual está fundamentado na educação inclusiva com habilidades diversas, que foram recolhidas nos anos 2010-2011, no Colegio Real de los Andes. Tal pesquisa esteve baseada na experiência pessoal da autora com esta população dentro do ensino regulamentar, motivada pelo desejo de conhecer como pode ser o desempenho destas pessoas, as quais devem ser olhadas pela sociedade em geral como inclusivas, e ainda mais quando se fala em termos de educação com um referente de formação integral, tendo presente que o ser humano é único e irrepetível, com potencialidades várias, grandes expectativas e sonhos. Isso envolve que seja um ator principal em um projeto de vida digno, que irá servir para o desenvolvimento pleno da sua personalidade e fortalecer suas habilidades acadêmicas. Por isso, é muito importante que o amor à inclusão seja parte essencial dos educadores como característica fundamental não somente do conceptual, mas, ainda mais, que deve ser assumida como parte fundamental da essência do educador, quem deve ser, segundo a minha opinião, um ser humano conformado com grande riqueza de valores, que deve se projetar em uma pedagogia com mentalidade aberta, livre de prejuízos e preconceitos, para assim conseguir pôr em prática sua pedagogia.

Palavras chave: inclusão, habilidades diversas, formação integral, Pedagogia da Integração, Colegio Real de los Andes, marco legal, integração. 
Esta investigación es el resultado del proyecto realizado entre los años 20102011, sobre la adaptación del currículo de un alumno en el aula regular, orientado a acopiar información y crear conciencia colectiva acerca de la igualdad de derechos. Las personas tienen capacidades diversas para aprender con igualdad de oportunidades, poder convivir y trabajar aceptando las diferencias individuales, teniendo buenas relaciones y logrando tener un sentido comunitario en el cual debe existir apoyo y una buena relación entre el educador y sus estudiantes. Todo ello se puede evidenciar en la Institución Educativa Real de los Andes, con un aprendizaje compartido en el que aun cuando se trabaja con el estudiante de manera individual, se le ve como parte de la colectividad, participando y socializando las diversas experiencias no solo orientadas a los niños, sino a la comunidad educativa en general: estudiantes, educadores, personal administrativo y familia. Este proyecto de investigación pretende dar algunas herramientas pedagógicas a los educadores, pues aunque ya existan las leyes, no se debe desconocer que, en nuestro país, respecto al conocimiento sobre la integración a la educación de los niños existen aún grandes falencias y desconocimiento por parte de muchos, incluso de los mismos educadores, esto según estudios de la Secretaría de Educación.

Los diferentes síntomas que presentan los niños y niñas con diferentes dificultades dentro del aula regular, en su mayoría se refieren concretamente al aprendizaje. Es un hecho que hoy en día existe un gran número de niños que presentan dificultades para su aprendizaje, hasta el punto de hacernos pensar en un aumento de alumnos-problema con relación a épocas anteriores. Aunque es cierto que se posee en la actualidad mejores técnicas de la pedagogía, debería existir una mayor sensibilización socioescolar y unos programas de enseñanza orientada a la realidad del niño, teniendo en cuenta las diferencias individuales con que el problema se produce en cada estudiante.

Si el niño y niña se encuentra con padres y educadores que adoptan posturas exigentes y rígidas ante el rendimiento escolar o que hipertrofian el valor que para este tienen las notas y calificaciones; no se les permite el error. Se olvida que la valoración del rendimiento académico es un escalón más en el proceso de aprendizaje. Esta situación de exigencia crea en gran número de niños una reacción negativa, y en lugar de superar las dificultades que naturalmente surgen ante el aprendizaje de unas técnicas inapropiadas, las aumentan y sobreviene el fracaso con todas sus secuelas psicológicas y sociales, alterando la vida y desarrollo del niño, de aquí, la gran importancia de la adecuada y oportuna integración de un niño con dificultades en el aula regular. Formulando esta situación como problema, se podría llegar a preguntar cómo sería la adaptación de un niño con habilidades diversas en el aula regular. Se delimita este problema de investigación realizada en el Colegio Real de los Andes de Chía, Cundinamarca, Barrio los Chilacos Cra 9 N. 21-171, fundado en 1997 por la señora Maribel Pardo Pedraza, representante legal de la institución. 
Para esta investigación se tomaron los estudiantes de preescolar hasta el grado de 5 de primaria, donde se incluirán los niños que tengan algún tipo de dificultad o discapacidad, ya sea mentales, problemas de aprendizaje, discapacidad intelectual, autismo, síndrome de Down, psicomotrices, parálisis cerebral y retardo en el desarrollo; sensoriales sordos, hipoacusicos, ciegos, baja visión. Este tipo de población se escogió para saber identificar las diferentes problemáticas y así, realizar una adecuada intervención pedagógica y didáctica que ayude a orientar nuestro saber profesional y pedagógico.

Para hablar de esta problemática se debe comenzar a describir qué se comprende como una dificultad o por el contrario este término solo es generalizado, así mismo, se establecerá si es una buena solución la adaptación de estos niños en el aula regular o si esto puede llegar a incrementar sus dificultades.

Nuestro espacio de investigación fue la institución Real Andes en la cual ingresan niños con dificultades que son integrados al aula regular. La observación fue la base de nuestra investigación, y el objetivo fue realizar un análisis descriptivo sobre la adaptación de un niño con dificultades en el aula regular. La revisión bibliográfica permitió adquirir conocimientos sobre los menores, con el fin de determinar los tipos de dificultades que se pudieran dar, haciendo una descripción de los métodos de intervención pedagógica que es posible trabajar con los niños.

Se indagó, también, sobre la importancia de las normas en el marco legal, que se deben tener con los niños y niñas, y saber cómo con la ley la podemos implementar, siendo un derecho para todos los estudiantes según la Constitución Política de Colombia, Ley General de Educación de 1994 y el Código de Infancia y Adolescencia. Estos estatutos se encargan de promover la educación para personas con habilidades diversas, para que puedan integrarse y ser partícipes de una educación con calidad, acorde a sus necesidades, sin olvidar que deben ser sujetos con derechos e igualdad de oportunidades y, más aún, cuando se está hablando en términos de educación, la cual se debe orientar a estimular por medio de la creación de proyectos educativos.

El desarrollo de la experiencia colombiana en la formación del talento humano para la implementación de la política de integración, se ha dinamizado a partir de la Constitución Política de 1991, la cual es novedosa para el país con sus planteamientos en la igualdad de oportunidades para el desarrollo, la participación de las personas en los procesos sociales, concepción del hombre como ser integral (como ser cultural e histórico) y en la necesidad de construir la nacionalidad. Posterior a la Constitución, la Ley General de Educación de 1994, promueve que la educación para personas con necesidades educativas se integra al servicio público educativo, hecho que genera un cambio en la estructura de este, a partir de esta Ley se concibe la educación en el país como un solo sistema que atiende a la diversidad de la población, fenómeno que invita a contextualizar la educación a través de los proyectos educativos institucionales. 
Políticas educativas posteriores a la Ley General de Educación, como el Plan Decenal de Desarrollo Educativo (1996 - 2005) y el salto educativo (1994 - 1998), programa de gobierno del actual presidente, promueven la equidad en el sistema educativo y sustentan la necesidad de programar la educación, teniendo en cuenta la pluralidad de la población. El Decreto 2082 de 1996, sobre Atención Educativa para Personas con Limitaciones o con Talentos Excepcionales, reglamenta los artículos 46 al 49 de la Ley General de Educación, para garantizar la integración escolar y social para estas personas en las Instituciones de Educación Formal y No Formal y en Programas Informales. De acuerdo con la legislación colombiana dispuesta en la ley de educación título III, capítulo I. La cual dispone: educación para personas con limitaciones o capacidades excepcionales. Artículo 46: integración con el servicio educativo la educación para personas con limitaciones físicas, sensoriales, psíquicas, cognoscitivas, emocionales o con capacidades intelectuales excepcionales, es parte integrante del servicio público educativo. Los establecimientos educativos organizaran directamente o mediante convenio, acciones pedagógicas y terapéuticas que permitan el proceso de integración académica y social de dichos educandos.

Las instituciones educativas que en la actualidad ofrecen educación para personas con limitaciones, la seguirán prestando adecuándose y atendiendo a los requerimientos de la integración social y académica, desarrollando los programas de apoyo especializado necesarios para la adecuada atención integral de las personas con limitaciones físicas, sensoriales, psíquicas, o mentales. Este proceso se realizara en un plan no mayor de seis años y será requisito esencial para que las instituciones particulares o sin ánimo de lucro puedan contratar con el Estado:

"Sé que en Colombia existen proyectos de educación inclusiva, pero son esfuerzos aislados que hay que replicar en todo el país", dice Porter, y asegura que los colegios privados podrían liderar ese gran paso a la inclusión, que él denomina "el camino hacia el futuro". (El Espectador, 2011)

En Colombia, la educación inclusiva de las personas con discapacidad es un modelo educativo reciente que supera los tradicionales modelos sobre los que se ha basado la educación. Precisamente porque representa un cambio en torno al derecho de educación. El modelo inclusivo ha generado resistencia por parte algunos funcionarios públicos, educadores. (Porter, 1991). Lo cierto es que la educación inclusiva, enunciada por Porter, no solo apuesta en Colombia, sino en el mundo entero. El artículo 24 de la Convención de las Naciones Unidas sobre los Derechos de las Personas con Discapacidad (CDPD), es el mejor ejemplo ello. En dicho artículo se materializa la obligación de garantizar un Sistema de educación inclusivo a todos los niveles, así como la enseñanza a lo largo de la vida, con miras a hacer posible que las personas con discapacidad participen de manera efectiva en una sociedad libre (Porter, 1991). 
Según estos planteamientos, Porter afirma:

"[...] la inclusión escolar significa que todos los alumnos, incluso aquellos que tienen discapacidades o alguna otra necesidad son escolarizados en aulas ordinarias, con compañeros de su misma edad y en escuelas de su comunidad [...]". Así mismo, en el marco de inclusión de escolar y desde esta perspectiva, todos los niños y niñas van a la escuela ya sea en su barrio o pueblo: "[...] los estudiantes con necesidades especiales van a la escuela donde ya irían si no fueran discapacitados y están en una clase comun con sus compañeros [...]" (Porter, 2001, p.7).

Asimismo. la UNESCO (2001), afirma que se pueden considerar inclusivos las escuelas, los centros de aprendizaje y los sistemas educativos que están abiertos a todos los niños y niñas. Estos centros se caracterizan por el intento de identificar cualquier barrera que pueda obstaculizar el aprendizaje de todos sus miembros y procuran reducirlas o eliminarlas (Padros, 2011). Por otro lado, Stainback (2001a, p.18) afirma que:

"[...] la educación inclusiva es el proceso que se ofrece a todos los niños y niñas, sin distinción de discapacidad, raza o cualquier otra diferencia, la oportunidad para continuar siendo miembro de la clase ordinaria y para aprender con sus compañeros y justamente con ellos dentro del aula [... $]^{\prime \prime}$.

Otros autores se enfatizan la idea que la inclusión escolar es un proceso de mejora de la escuela que tiene por objetivo eliminar los procesos de exclusión. En este sentido, consideran que el proceso de inclusión tiene por objetivo identificar y responder a la diversidad de necesidades de todos y todas las estudiantes a través de una mayor participación en el aprendizaje (Ainscow, 2005; UNESCO, 2005).

Por otro lado, encontramos más allá de argumentos éticos, los basados en los beneficios de la educación inclusiva para todas las personas que forman parte de la comunidad educativa, así mismo la eficiencia de las escuelas que incluyen a todos los niños y niñas en sus aulas. En este sentido, las investigaciones apuntan que separando a los alumnos y alumnas de forma homogénea no mejora todo el conjunto. De hecho, los y las estudiantes con baja capacidad tienen los peores resultados en estas situaciones que cuando están asignados en grupos heterogéneos (Gimeno Sancristan, 2005, p.32). 


\section{Aspectos teóricos}

\section{Pedagogía de la integración}

Desde la aparición de la nueva Constitución y de las demás normas y políticas derivadas de ella, se evidencia que la formación inicial y continua de maestros, debe tener una profunda transformación para superar las barreras latitudinales, las rutinas institucionales y la práctica docente en su quehacer pedagógico. Se genera en el país el debate en torno al qué y el cómo formar la nueva generación de maestros.

Considero que la integración es una filosofía que rodea la vida cotidiana y que dignifica al hombre, pero que su valoración se ve afectada por las circunstancias que ocurren al interior de una cultura y de una sociedad. Para construir una teoría para la integración se requiere del abordaje de concepciones al nivel cognitivo, funcional y emocional de las comunidades educativas con relación a las personas con necesidades educativas:

Desde la concepción cognitiva, implica aproximarse al conocimiento de las necesidades educativas a partir de las teorías sobre procesos de desarrollo y aprendizaje del niño. Los escenarios donde se desenvuelve la persona con necesidades especiales son un espacio ambivalente, contradictorio de desigualdades pero también de oportunidades, que para enfrentarlos implica comprender el proceso cultural del grupo y poder actuar acorde al contexto.

Cuando se habla de integración como filosofía de vida, se parte del hecho que creo en el otro como un ser integro tal cual es en saberes, creencias, potencialidades y necesidades que para impulsar su desarrollo, se requiere consolidar estrategias pedagógicas y ambientales, se pretende que la comunidad educativa se apropie del saber generado por investigaciones y experiencias significativas en integración para una mayor comprensión de esta política educativa.

Desde la concepción funcional se interpreta que el concepto de necesidades especiales implica estrategias, recursos y adaptaciones curriculares y especialmente el cómo orientar una institución.

Teniendo presente que la población objeto de estudio cumple con los requisitos para el abordaje del tipo de educación integral, las investigadoras consideran pertinente dar una mirada general a los principales tipos de dificultades que se pueden presentar dentro del aula: 


\section{Dificultades cognitivas}

El niño puede tener un desarrollo más lento en las áreas:

1. Desarrollo conectivo

2. Desarrollo de lenguaje

3. Coordinación motora

El niño puede tener dificultades para aprender habilidades nuevas, para que el niño pueda aprender una labor o habilidades nuevas son necesarias muchas horas de práctica, el niño puede darse por vencido o perder el interés cuando la actividad es muy difícil, el niño puede ser:

1. Amigable o retraído

2. Impulsivo o tímido

3. Difícil de entregarse a algo

Esta categoría puede incluir niños con discapacidad intelectual o síndrome de Down.

\section{Problemas de aprendizaje}

Los problemas del aprendizaje afectan a 1 de cada 10 niños en edad escolar. Son problemas que pueden ser detectados en los niños a partir de los 5 años de edad y constituyen una gran preocupación para muchos padres ya que afectan al rendimiento escolar y las relaciones interpersonales de sus hijos.

\section{Déficit de atención e hiperactividad}

Es la ausencia, carencia o insuficiencia de actividades de orientación, selección o mantenimiento de la atención, así como la deficiencia del control, su participación con otros procesos psicológicos con sus consecuencias específicas desde el punto de vista neurológico. Se asume que el déficit de atención es un trastorno de función cerebral en los niños adolescentes y adultos, caracterizado por la presencia persistente de síntomas comportamentales y cognoscitivos de deficiencia atencional, interactividad e hiperactividad.

\section{Hiperactividad}

La hiperactividad es un trastorno de la conducta en niños. Se trata de niños que desarrollan una intensa actividad motora, que se mueven continuamente, sin que 
toda esta actividad tenga un propósito. Van de un lado para otro, pudiendo comenzar alguna tarea, pero que abandonan rápidamente para comenzar otra, que a su vez, vuelven a dejar inacabada. Esta hiperactividad aumenta cuando están en presencia de otras personas, especialmente con las que no mantienen relaciones frecuentes. Por el contrario, disminuye la actividad cuando están solos.

\section{Discapacidad intelectual y síndrome de Down}

Es una afección diagnosticada antes de los 18 años de edad, que incluye un funcionamiento intelectual general por debajo del promedio y una carencia de las destrezas necesaria para la vida diaria. La discapacidad intelectual es la presencia de un coeficiente intelectual bajo que se vuelve un problema considerable en la adaptación de la vida diaria, presentando alteraciones a nivel orgánico, psíquico, social y cognitivo. Tal discapacidad se caracteriza por un funcionamiento inferior a la media, junto con limitaciones asociadas en dos o más de las habilidades adaptativas de comunicación y cuidado personal, vida en el hogar, habilidades sociales que se utilizan en la comunidad, salud y seguridad, habilidades académicas funcionales, sociales y de trabajo.

\section{Síndrome de Down}

Es un trastorno genético causado por la presencia de una copia extra de cromosomas o una parte del mismo, en vez de los dos habituales, trisomía del par, caracterizado fisicamente por la presencia de un grado variable de trastorno mental y unos rasgos físicos peculiares que le dan un aspecto reconocible.

\section{Trastornos del espectro autista}

Es un trastorno infantil que suele darse más en los niños que en las niñas y su mayor compromiso se encuentra en la socialización y a nivel comunicativo, presentándose un déficit de los mismos. Un niño autista puede puede o no desarrollar todo tipo de habilidades, dependiendo tanto del nivel de coeficiencia intelectual y la capacidad de comunicación verbal.

\section{Discapacidad motora}

Los trastornos del desarrollo psicomotor son muy difíciles de definir, reflejan siempre alteraciones en las que se ven afectados varios aspectos del desarrollo del niño, de ahí la importancia de intervenir cuanto antes, pues el trastorno puede ir repercutiendo negativamente en otras áreas de la vida del niño, agravando y comprometiendo su desarrollo. 


\section{Parálisis cerebral}

Parálisis cerebral es un trastorno que afecta a miles de bebés y niños cada año. No es contagiosa, lo que significa que no puedes contraerla de una persona con parálisis cerebral. Es una debilidad o problema en la manera en la que una persona mueve o posiciona su cuerpo. Una persona con parálisis cerebral tiene dificultades para controlar los músculos del cuerpo. Normalmente, el cerebro le dice al resto del cuerpo exactamente qué hacer y cuándo hacerlo. Pero como la parálisis cerebral afecta al cerebro, dependiendo de la parte del cerebro afectada, el niño podría no poder caminar, hablar, comer o jugar de la manera que lo hace la mayoría de los niños.

\section{Discapacidad visual}

\section{Niños ciegos}

El papel de la sociedad frente a un niño con discapacidad es sumamente importante para que el niño se sienta integrado y respetado. Un niño, con algún impedimento, bien atendido, educado y aceptado podrá hacer las misma cosas que cualquier otro niño, solo que de un modo muy distinto. La actitud de respeto debe empezar desde el momento que se detecte la discapacidad, no hay que limitar sus posibilidades por la simple razón que el niño tenga alguna discapacidad, al contrario hay que animarlo y enseñarle las cosas por las cuales podrá hacer lo que desee, por ejemplo: no sería aconsejable que un niño ciego cruce la calle sin antes haber recibido la orientación, pero no se debe pensar que un niño ciego jamás podrá cruzar la calle por él mismo.

\section{Discapacidad auditiva}

\section{La hipoacusia}

En los niños puede ser congénita y adquirida, la sordera puede ser congénita por causas neuro sensoriales, esta a su vez puede ser de origen retro codear lesión desde la cadera al corte cerebral, puede ser también de transmisión, esta puede ser por malformación del oído medio o externo o por problemas de oído medio; de causa transitoria como es la otitis serosa esta se puede mantener de forma permanente pero sin detección., La hipoacusia es la disminución del nivel de audición por debajo de lo normal, lo cual constituye un motivo habitual de consulta y es especialmente frecuente en la población anciana, oscilando entre el $25 \%$ en los mayores de 65 años y el $80 \%$ en los mayores de 80 . Con frecuencia, da lugar a situaciones de minusvalía con importantes repercusiones físicas y psicológicas. 
Según su intensidad, la hipoacusia se clasifica en:

Leve (pérdida menor de $35 \mathrm{~dB}$ ),

Moderada (pérdida entre 35 y $60 \mathrm{~dB}$ ),

Profunda (pérdida entre 60 y $90 \mathrm{~dB}$ ) y

Total o cifosis (pérdida superior a $90 \mathrm{~dB}$ )

Hay que diferenciar las hipoacusias neurosensoriales o de percepción (por lesiones en la cóclea, en las vías neuronales o en el sistema nervioso central, en la corteza auditiva) de las de transmisión o de conducción (por alteraciones del oído externo o medio que impiden la transmisión normal del sonido) y de las formas mixtas.

El desarrollo de la experiencia colombiana, con la formación del personal humano para la implementación de la política de integración, se ha dinamizado a partir de la Constitución Política de 1991, la cual integra en sus planteamientos la igualdad de oportunidades para el desarrollo personal en procesos sociales y culturales posterior a la constitución, la ley general de educación de 1994, promueve de la misma manera dichos planteamientos. Legislación de gran importancia porque se refiere a cómo, a través de las experiencias vividas, podemos observar que la adaptación de estos niños en un aula regular logra un proceso integral. De esta forma, todos se sienten incluidos, ya que este tipo de educación consigue que todos los estudiantes aprendan a su ritmo y logren una buena formación. ,A partir de un aprendizaje personalizado, donde el maestro debe formarse para que pueda generar nuevas estrategias y alternativas inclusivas dentro del aula. Porque al tener niños con habilidades especiales en un salón de clase, muchas veces se les deja en un rincón e incluirlos es muy necesario para un aprendizaje significativo, propiciando socialización y potenciando sus habilidades. Sin embargo, su mayor dificultad es hacer una educación inclusiva, se dice que es un sistema que tiende a estandarizar las formas de enseñanza y evaluación, pues quieren que todos los niños sean iguales y cuando hay niños que expresan diferencias y debilidades son vistos como problema, no como una oportunidad de transformación pedagógica,(Pabón, 2010). Algunos otros autores también señalan la importancia de la educación inclusiva, como María Montessori que empezó enseñando y apoyando a niños con habilidades especiales desde sus diferencias.

El resultado de este proyecto se debe a una metodología cualitativa, que tiene como objeto la investigación descriptiva de las cualidades de un fenómeno, y que busca un concepto que pueda abarcar parte de una realidad, no se trata de probar o de medir en qué grado de cierta cualidad se encuentra un cierto acontecimiento dado, sino de descubrir tantas cualidades como sea posible. En investigaciónes cualitativas, se debe hablar de entendimiento de profundidad en lugar de exactitud, se trata de obtener un entendimiento lo más profundo posible. El interés de este paradigma es centrarse en las relaciones humanas y la vida social (Erikson, 1986). 


\section{Métodos Cualitativos}

En el estudio de casos podemos indicar, según Cohen (1990: 164), que:

Si bien el experimentador maneja variables para determinar su significación causal
o el encuestador hace preguntas normalizadas a grandes y representativas muestras
de individuos, por el contrario, el investigador del estudio de casos observa las
características de una unidad, un niño, una pandilla, una escuela o una comunidad.

\section{Análisis interpretativo}

Estudios descriptivos: Ios estudios descriptivos seleccionan una serie de cuestiones y se mide cada una de ellas en forma independiente. Desde el punto de vista científico, describir es medir. Por ejemplo, un investigador organizacional pretende describir varias empresas industriales en términos de su complejidad, tecnología, tamaño, centralización y capacidad de innovación. "Los estudios descriptivos buscan especificar las propiedades importantes de personas, grupos, comunidades o cualquier otro fenómeno que sea sometido a análisis" (Dankhe, 1986).

Fase proactiva: En ella se tienen en cuenta los fundamentos epistemológicos que completan el problema o caso, los objetivos pretendidos, la información de que se dispone, los criterios de selección de los casos, las influencias del contexto donde se han desarrollado los estudios, los recursos y las técnicas que se han de necesitar. En esta fase, también se considera muy importante la temporalización prevista y su relación con el seguimiento que ha de hacerse de la investigación proyectada.

Fase interactiva: corresponde al trabajo de campo y a los procedimientos y desarrollo del estudio, utilizando diferentes técnicas cualitativas: tomas de contacto y negociación que sirven para delimitar las perspectivas iníciales del investigador, las entrevistas, la observación y las evidencias documentales. De cualquier modo, en esta fase es fundamental el procedimiento de la triangulación para que pueda ser contrastada la información desde fuentes diferentes.

\section{Educación personalizada}

El resultado es una educación con programas de educación personalizada, PEP, que se establecen con el plan de estudios y la descripción de todas las actividades de los niños con dificultades o necesidades educativas especiales.

¿Cómo debe ser la actitud del maestro para la adaptación con un niño con dificultades en el aula regular? La respuesta a esta pregunta, más allá de la interacción maestro-estudiante y de la utilización de materiales especializados, se encuentra en el hecho de que estos niños se sientan queridos aceptados y escogidos como amigos, se habla de una interacción con una influencia positiva tanto de los estudiantes 
que tienen dificultades, como de los que no las tienen. Al integrar a los niños al aula regular, la mejor forma para adaptarlos es promover la oportunidad de crear y desarrollar relaciones benéficas tanto de los estudiantes con dificultades, como los que no las tienen, generando relaciones verdaderas de amistad, algo que influye en el mejoramiento cognitivo y capacidad de progreso, en la medida que van avanzando por diferentes niveles. Los niños se desarrollan según su propio ritmo de aprendizaje. Es decir, que los estudiantes con dificultades no se puede debe decir que perdieron un año lectivo, si no, que avanzan progresivamente de acuerdo con sus capacidades, habilidades, destrezas, y ganancias en su aprendizaje, nivel cognitivo y desarrollo socioemocional.

Inicialmente, los niños que ingresan al colegio Real de los Andes se les hace una valoración inicial respecto a su desempeño académico y dependiendo de dichos resultados son ubicados en un aula especializada adaptada a las necesidades especiales de cada niño, es decir, se trabaja de manera personalizada y según sus logros y nivel de madurez, avanzan hacia otro nivel superior para pasar de manera gradual con los estudiantes que no tienen dificultades desde el principio del proyecto educativo. El educador debe ser preparador de lo que significa para todos la integración de los niños con o sin dificultades, tratando al máximo de minimizar las diferencias que existen, mediante la práctica del respeto mutuo, la tolerancia y una sana convivencia, esta es una de las mayores funciones del maestro.

En Colombia ya se están dando pasos importantes para la inclusión de las personas en situación de discapacidad a las aulas regulares, la cual debe incluir proyectos articulados al PEI que den respuesta a las necesidades de este tipo de población. Por ello es importante que las personas que nos estamos formando como docentes, veamos desde ahora el punto de vista positivo de la inclusión de niños con alguna limitación, que se entienda no como una obligación, sino como una oportunidad.

La inclusión de los niños con discapacidades es el ideal a alcanzar, aunque aún se debe aprender a aceptar las diferencias, algo que aún en nuestros días es difícil de aceptar por la sociedad y a veces por la misma familia. Por esta razón, se requiere tener una mente más abierta, realizando trabajos con las familias, profesores y en general con toda la comunidad para que el resultado sea el esperado y estos niños puedan tener un lugar donde se sientan felices y no discriminados como suele ocurrir, entonces es importante que desde los jardines, colegios y universidades se tenga un currículo flexible que se adapte a las condiciones y al contexto de las personas en situación de discapacidad, sin importar cuál sea esta.

Los niños integrados aprenden más y mejor con el estímulo de sus compañeros y de un docente amable que los quiera y los respete, también es importante que los maestros aprendan a reconocer sus capacidades y habilidades. Es una cuestión de sensibilizarnos y actuar sin prejuicios, viendo dicha experiencia como algo 
que puede ser un gran aprendizaje para nuestras vidas, también es una oportunidad importante para fortalecer valores como el respeto a la diferencia. Las personas en situación discapacidad, pueden aprender a ser autónomas e independientes, superando sus dificultades. La inclusión de los alumnos con discapacidad debe ser un proceso que requiere de importantes progresos a nivel teórico y práctico, donde los profesores debemos aprender a implementar una serie de medidas y acciones específicas, para atender las necesidades educativas de este grupo particular de estudiantes.

Las niñas, niños y adolescentes en situación de discapacidad deben desarrollarse en sus áreas de mayor potencial, es por ello por lo que, debemos poner en práctica una pedagogía y una evaluación diferenciada para cada alumno, tras puntualizar que la educación debe ser cada día más humanista y moderna. Viendo al ser humano de una manera global, no solo como la persona que adquiere conocimientos y aprendizajes de tipo académico, sino lo más importante, aprendizajes para un buen vivir. Estos niños deben estar en un lugar donde se sientan queridos, aceptados y respetados por todos, y sobre todo, por los educadores.

En Colombia ya se están dando pasos importantes para la inclusión de las personas en situación de discapacidad a las aulas regulares, estos intentos deben incluir proyectos articulados al PEI que den respuesta a las necesidades de este tipo de población. Por ello es importante, que las personas que nos estamos formando como docentes tomemos en cuenta la inclusión de niños con alguna limitación y que se entiendan, no como una obligación sino como una oportunidad. Este es el ideal a alcanzar, aunque aún se debe aprender a aceptar las diferencias, algo que aún en nuestros días es difícil de aceptar por la sociedad.

¿Cómo debe ser la actitud del maestro para la adaptación con un niño con dificultades en el aula regular? La respuesta a esta pregunta, más allá de la interacción maestro-estudiante y de la utilización de materiales especializados, es que cuando estas personas se sienten queridas y aceptadas hay una interacción con una influencia positiva, tanto de los estudiantes que tienen dificultades, como los que no. El integrar a los niños al aula regular es la mejor forma para adaptarlos, influyendo así en promover posibilidad de crear y desarrollar relaciones benéficas entre todos los estudiantes, consolidanto las relaciones de amistad y esto de alguna manera influye sobre el mejoramiento cognitivo y capacidad de progreso, y así lograr ir avanzando en los diferentes niveles. Lo anterior sucede según su propio ritmo de aprendizaje, es decir, que los estudiantes con dificultades no se podría decir que pierden un año lectivo.

Las autoras observaron que los niños con dificultades se pueden adaptar en el aula regular dependiendo su ritmo de aprendizaje, es decir, de acuerdo con el nivel de habilidades y capacidades que posean, ya sean cognitivas, socio afectivas y comunicativas. La mejor forma de adaptación es la integración de todos los estudiantes en general, ya que gracias a ello, se aprende a superar las diferencias, aceptándose y 
tolerándose, sin importar si tienen o no algún tipo de dificultades, esto mejora las relaciones del grupo y por ende, su proceso de enseñanza-aprendizaje. A los niños se les realiza una valoración y dependiendo del resultado se les ubica en un aula especializada de manera personalizada.

Pautas que se tienen presentes:

La familia deberá

- Tener normas claras y bien definidas.

- Dar órdenes cortas y de una en una.

- Propiciar un ambiente ordenado, muy organizado, sereno y sin gritos.

- Reconocer el esfuerzo realizado por el niño.

- Evitar ser sobreprotectores y no dejarse influenciar por los caprichos de los niños.

- Cumplir siempre los castigos y las recompensas ante sus acciones.

- Darle al niño pequeñas responsabilidades.

- Aceptar al niño tal y como es.

- Saber que el trabajo es mucho y que se necesita mucha constancia.

- Fomentar los puntos fuertes, y las facultades del niño.

\section{Conclusiones}

Podemos concluir que el modelo de pedagogía de la integración, centra su atención en la diversidad, aspecto que ha tenido muchos cambios a lo largo de los años. Hace muchos siglos, se pensaba que estas personas eran creadas por seres malignos, demoníacos, y entonces, eran encerrados e incluso sacrificados. Posteriormente, se crearon centros especializados para atender a este tipo de estudiantes en particular, con un currículo diferente al del alumnado escolarizado en centros ordinarios. En los años sesenta, con la intención de que las personas con algún tipo de deficiencia aprendieran por imitación y que aumentaran la influencia entre especialistas y profesores, todo esto para desarrollar actitudes de respeto y solidaridad entre los otros alumnos, y que en general, la sociedad se volviese más tolerante y abierta. Todo esto para preparar a estos sujetos para afrontar una sociedad competitiva, como en la que vivimos actualmente, y proporcionarles un entorno escolar para vivir con unas condiciones y formas de vida lo más normalizadas posible (López, 2011). 
Posteriormente, también entrarían en juego las teorías de las inteligencias múltiples de Gardner (1983), teniendo en cuenta el diverso desarrollo de cada sujeto en cada una de estas, además de la inteligencia interpersonal e intrapersonal, para favorecer su desarrollo. (Pérez Sánchez, 2007) Este investigador y pedagogo afirma que a nivel general, el sistema de enseñanza actual, al igual que los métodos para evaluar, solo se recurre a algunos tipos de inteligencia como son la verbal y la matemática. Él ha identificado no solo una, sino diferentes modalidades de inteligencia, descritas en su libro de Frames of Mind (las estructuras de la mente), la inteligencia racional y matemática, (lógica) visual, auditiva y verbal (lingüística), musical y rítmica, kinestésica (física), intrapersonal, interpersonal y natural (Pardo, 2002, p. 2).

Existen suficientes pruebas positivas de que algunas personas adoptan una aproximación inmediata al aprendizaje, mientras que otras prefieren un rumbo especial o cuantitativo. Igualmente, algunos estudiantes obtienen los mejores resultados cuando se les pide que manejen símbolos de clases diversas, mientras que otros están mejor capacitados para desplegar su comprensión mediante demostraciones o a través de la interacción con individuos (Pardo, 2002, p.10). Gardner ha postulado que todos los seres humanos son capaces de conocer el mundo de modos diferentes y estos modos la ha llamado, las inteligencias humanas, estas desafían un sistema educativo que supone que todo el mundo puede aprender las mismas materias del mismo modo, y que basta con una medida uniforme y universal para poner aprueba el aprendizaje del estudiante (Pardo, 2002, p.11). Investigación muy importante, ya que es una herramienta fundamental como base curricular y modelo. Sin dejar atrás la educación integral y personalizada, que junto a la teoría de la inteligencias multiples son la base para el mejor aprendizaje de los estudiantes con habilidades diversas.

Según la investigación observada en este artículo, los niños que pertenecen al Colegio Real de los Andes son un grupo estudiantes en el que prima la integración, existe una mirada más amplia en los diferentes contextos, y un trabajo armónico y coordinado con la institución y su familia. Es así, que en la institución todos se sientan queridos, aceptados e integrados a la comunidad educativa, es decir, no existen grandes diferencias entre aquellos niños que tienen alguna dificultad, con las que no la tienen. El aprendizaje para los educadores debe ser vivido, sentido, actuado e incorporado a sus prácticas y saberes profesionales de una manera donde existan espacios de sana convivencia, diálogo y participación de todos sus estudiantes. Los profesores no deben, por ningún motivo, discriminar a los niños, sino todo lo contrario, formarlos en valores y creando las posibilidades de desarrollar al máximo las capacidades de los estudiantes.

Si se logra una adecuada educación, los niños mejoran sus niveles de aprendizaje, desempeño y funcionalidad, tanto el colegio, como en el hogar, brindándoles 
métodos de enseñanza acorde a sus capacidades, donde radica la verdadera importancia de una educación personalizada, respetando sus diferentes ritmos de aprendizaje. De tal manera, que exista un grupo de aula especializada donde el desarrollo de sus actividades se realiza acorde al nivel de sus diferentes habilidades. Otros son los niños que han superado gradualmente sus dificultades y son integrados al aula regular, pero en términos generales, debe primar el respeto, la tolerancia y la cooperación, por parte de todas las personas (padres, educadores, directivos, estudiantes).

Finalmente, con estas reflexiones se pretende que el resultado de la investigación nos genere un cambio para actuar de manera más abierta, así como lo contemplan: la Constitución Política, la Ley General de Educación y el Código de la Infancia y Adolescencia, que buscan el desarrollo pleno de la personalidad y del ser humano, brindando amor, comprensión, disfrutando de los derechos, y también respetando y acatando los deberes. 


\section{Referencias}

Andrade, M. (2000). Influencia de las inteligencias múltiples, el rendimiento académico previo y el currículo del hogar sobre la autoestima de los alumnos de II Medio de la comuna de Santiago. Tesis de Grado para Doctorado en Ciencias de la Educación. Pontificia Universidad Católica de Chile: Santiago de Chile, Chile

Congreso de Colombia. (8 de febrero de 1994). Ley General de Educación. [Ley 115 de 1994]. DO: 41.214 .

Constitución política de Colombia. (1991) 2da Ed. Bogotá: Legis

Lenenberg, E. (1967). Fundamentos biológicos del infante. Alianza: Madrid.

López, M. (2005). Educación, amor, ética... caminos para construir un sueño: la escuela inclusiva. Revista de Ciencias de Educación. 21, 11-27.

Ministerio de Educación Nacional. (18 de Noviembre de 1996) Por el cual se reglamenta la atención educativa para personas con limitaciones o con capacidades o talentos excepcionales. [Decreto 2082 de 1996]

Organización Mundial de la Salud. (1992). Trastornos mentales y de comportamiento: Descripciones clínicas y pautas para el diagnóstico. Madrid: OMS.

Parra, M., Pérez, Y., Torrejón, M., Mateos, G. (2010). Asesoramiento educativo para la formación docente en la visión de escuela inclusiva. Revista Intercontinental de Psicología y Educación. 12(1), 77-87.

Parrilla, A., Moriña, A. (2004). Lo que todos nos preguntamos sobre la educación inclusiva. Padres y Maestros. 284, 10-14.

Pichot, P. (1995). DSM IV. Manual de diagnóstico y estadístico de los trastornos mentales. Barcelona: Masson.

Procuraduría General de la Nación. (2010). Código de la Infancia y la Adolescencia. [Ley 1098 de 2006]. Bogotá: Visión Mundial.

Ramírez. (2001)..Métodos de enseñanza del aula regular e integración a los niños con dificultades en el aprendizaje.

Robert, S. (2008). Desarrollo de la infancia. México: Prentice Hall.

Pardo, M. (2002) Experiencia del Colegio Nuevos Horizontes de Chía (Cundinamarca). Basado en el marco de las inteligencias múltiples.

Rosa, G. (2006). La equidad y la inclusión social: uno de los desafíos de la educación y la escuela hoy. Revista Electrónica Iberoamericana sobre Calidad, Eficacia y Cambio en Educación. 4,(3).

Sarramona, J., Rodríguez, T. (2010). Participación y calidad de la educación. Aula Abierta. (38)1, 3-14. 
UNESCO. (2005). Guidelines for inclusion: ensuring access to education for all. París: UNESCO.

Recibido: 2 de octubre 2014

Aceptado: 23 de noviembre 2014

Como citar:

Rodriguez, C.L.(2015)Adaptación del currículo de un alumno en el aula regular. Praxis Pedagógica, 16, 107-125. 